\title{
REGULARITY ISSUES FOR SEMILINEAR PDE-S (A NARRATIVE APPROACH)
}

\author{
H. SHAHGHOLIAN
}

Dedicated to Nina Nikolaevna Ural'tseva

\begin{abstract}
Occasionally, solutions of semilinear equations have better (local) regularity properties than the linear ones if the equation is independent of space (and time) variables. The simplest example, treated by the current author, was that the solutions of $\Delta u=f(u)$, with the mere assumption that $f^{\prime} \geq-C$, have bounded second derivatives. In this paper, some aspects of semilinear problems are discussed, with the hope to provoke a study of this type of problems from an optimal regularity point of view. It is noteworthy that the above result has so far been undisclosed for linear second order operators, with Hölder coefficients. Also, the regularity of level sets of solutions as well as related quasilinear problems are discussed. Several seemingly plausible open problems that might be worthwhile are proposed.
\end{abstract}

\section{$\S 1$. Elliptic CASE}

1.1. Background. This paper should be viewed as a laid-back approach to pointwise regularity of solutions of elliptic problems where there is a lack of sufficient regularity of the ingredients, which would otherwise have forced regularity of solutions. In particular, the current note should be seen as a roadmap to FB-regularity 11 in a nutshell, without too much details of technical analysis; see Remark 1.1

For clarity of exposition, and for the purpose of pointing out peculiarities in the theory, I have chosen to discuss most simple cases; e.g., I shall deal mainly with the Laplace equation, or very simple divergence type equations. It should also be stressed that all the discussions, but probably not all the derivations of the results, can be done for very general equations; I shall point this out later. And of course, with some perseverance, any astute reader will be able to make his/her own generalizations of the ideas in this note.

Since I shall deal with the local regularity of solutions, all equations can be considered in the unit ball $B_{1}$ (or unit cylinder for the parabolic case) without any reference to the boundary value. Also all statements about regularity shall be uniform in the half-ball $B_{1 / 2}$, where the norms depend on the supremum norm of solutions in the unit ball, as well as on all other ingredients, such as dimension, the operator, etc.

To set the scene, I depart from the one-dimensional case and recall the fact that if $u$ solves the ODE

$$
u^{\prime \prime}=f(x, u)
$$

2010 Mathematics Subject Classification. Primary 35J61, 35K58.

Key words and phrases. Pointwise regularity, Laplace equation, divergence type equations, free boundary problems.

Supported in part by Swedish Research Council.

${ }^{1}$ Here FB stands for Free Boundaries, but with the reservation that I consider only a certain class of problems, where there is a representation as a semilinear problem $\Delta u=f(u)$. 
with $f$ being bounded/continuous, then (by integration) $u$ must have bounded/continuous second derivatives. This naturally fails when one deals with PDE-s. Indeed, if $u$ solves

$$
\Delta u=f(x, u)
$$

with $f$ merely bounded, or even continuous, then one cannot expect $u$ to have bounded second derivatives. This can be seen in the following cute example.

A Cute Example. The function

$$
u(x)=x_{1} x_{2}[-\log |x|]^{a}, \quad 0<a \leq 1, \quad|x|<1,
$$

has bounded Laplacian, for $a=1$, and a continuous Laplacian for $0<a<1$, but the function is not $C^{1,1}$. One can also complicate this example, by replacing $\log |x|$ with several layers of log, i.e., slow down the singularity, but one cannot make the Laplacian to have Dini modulus of continuity 2

In dimensions larger than one, there is an extra (sufficient, but not necessary) condition for the right-hand side — the so-called Dini continuity — that plays a crucial role for the regularity theory.

The sufficiency of Dini continuity can be seen from a potential-theoretic approach, by using straightforward differentiation and then calculation of the integral (which is a very nice exercise for graduate students). The fact that Dini continuity is not always necessary can be seen by simply considering the one-dimensional case, and combining it with a second direction $u(x)=x_{1} H\left(x_{2}\right)$, where $H^{\prime \prime}=h$, with $h$ merely continuous, but not Dini. One may even complicate this by looking at $u=x_{1}\left(x_{2}^{+}\right)^{2}$, which is $C^{1,1}$ but has a discontinuous Laplacian.

When the right-hand side $f$ depends on $x$ only, then it seems that one has hit the end of the road for the regularity of the second derivatives. In other words, without Dini condition one cannot make any general statement about $D^{2} u$. There are, however, a plenty of situations when $f(x, u)$ is smooth in $x$ (or independent of $x$ ), but less regular in the $u$-variable, and the solution still exhibits enough regularity for its second derivatives.

My main objective with this note is to discuss particular cases when $f(u)$ has a structure (other than Dini) that enforces $D^{2} u$ to be bounded.

Remark 1.1 (For nonexperts). If new to the subject, the reader should consult the book PSU] before reading this note. The standard notation, concepts and methodology of the book [PSU] are frequently used in this note. Therefore, the current note is by no means self-contained, and is written to serve as a fast track to how the regularity theory works in free boundary problems. Nevertheless, the difference with the classical setting is that I consider general semilinear problems whose level-surfaces at a jump discontinuity for the right-hand side give rise to a free boundary, since there is a qualitative change in the PDE across such a level-surface.

1.2. Conditions for optimality. As a starting point, and for the simplicity and clarity of exposition, I shall consider the case of $f$ being independent of the $x$-variable:

$$
\Delta u=f(u) .
$$

The question one can ask is: what regularity should $f(u)$ have, in order to enforce bounds/ continuity on $D^{2} u$. E.g., it is well known (see $[\mathrm{Sh}$ ) that if $f(u)$ is Heaviside's function then $u$ is $C^{1,1}$. The result in $\left[\mathrm{Sh}\right.$ is actually slightly stronger, and states that if $f^{\prime} \geq-C$ for some constant $C$, then the second derivatives of $u$ (solutions of (10) are bounded. This result is a consequence of a monotonicity function, due originally to $\mathrm{H}$. Alt, L. Caffarelli,

${ }^{2} \mathrm{~A}$ function $h$ is Dini continuous at the origin if $\int_{0^{+}} h(t) / t$ is bounded. 
and A. Friedman $[\mathrm{ACF}$, that was developed for studying free boundary-value problems. See also CJK, MP, EP, TZ for further developments.

During the writing of this paper I realized that the proof in Sh actually has much stronger implications than stated in that paper, and therefore I shall formulate it as a new result (even though the proof is an observation) and leave the details out.

Theorem 1.2. Let $u \in W^{2, p}\left(B_{1}\right)(p>n)$ solve

$$
\Delta u=f(u) \text { in } B_{1} \text {, }
$$

with $f^{\prime} \geq-C$. Then for any point $z \in B_{1 / 2}$ and any directional vector $e \perp \nabla u(z)$ we have

$$
\lim _{r \rightarrow 0} \frac{1}{r^{n}} \int_{B_{r}(z)}\left|\nabla D_{e} u\right|^{2} \leq C^{\prime}\left\|\nabla D_{e} u\right\|_{L^{2}\left(B_{1}\right)}^{2}
$$

In particular, at any point $z,\left|D_{e} u(x)-D_{e} u(z)\right| \leq C^{\prime \prime}|x-z|$ for all $e \perp \nabla u(z)$ and $x \in B_{1 / 2}$. If $\nabla u(z)=0$, then $|\nabla u(x)-\nabla u(z)| \leq C^{\prime \prime}|x-z|$.

Remark 1.3. Actually the theorem applies to a larger class of function, with certain structure to guarantee that $\Delta\left(D_{e} u\right)^{ \pm} \geq-C$. For instance, if $f(u)=H(|u|)$ or $f(u)=$ $H(|\nabla u|)$, the results here still hold; see $[\mathbf{S h}$. What is more interesting is that these examples are nonvariational, and therefore the classical techniques are doomed to fail for this type of problems.

It might be possible to relax the condition $p>n$, but this is more related to having a monotonicity argument to work (see, e.g., [MP, Theorem III]). The theorem suggests that when the regularity breaks down, it does so in the direction orthogonal to the levelsurfaces. At the points where the derivatives of $u$ vanish, the function is formally $C^{1,1}$, even if $f$ is not bounded. Obviously, if $f \in L^{\infty}$, then the above theorem implies that the second derivatives are bounded at all points 3

In general, the above regularity theory breaks down if one allows $f^{\prime}$ to have a negative Dirac mass. This was cleverly demonstrated by an example of the form $f(u)=-\chi_{\{u>0\}}$, through a partially technical construction by J. Andersson and G. Weiss AW1.

The next question to raise is when can one expect continuity of the second derivatives? I.e., whether the continuity of $f(u)$ in $u$ implies the continuity of $D^{2} u$ ? This naturally fails if $f$ depends on $x$, and is merely continuous. Let us consider the following example:

$$
f(u)=\frac{-1}{\log u} \text { for } 0<u<1 / 2 .
$$

Then $f^{\prime} \geq 0$, and hence, by $\left[\mathbf{S h}, D^{2} u\right.$ is bounded. It is however not clear to me whether $D^{2} u$ is continuous, just because $f$ is also continuous.

One can naturally play around with several similar examples, which to my best knowledge has not been studied; e.g., what can we say about the regularity of solutions of the equation with $f(u)= \pm 1 / \log |u|$ ?

Observe that this function is not Dini. It is also obvious that if $f(u)$ is Dini continuous, then one obtains the continuity of $D^{2} u$ in a classical way. Hence, the question is whether one can push the condition below Dini in the semilinear case to obtain continuity of second derivatives.

\footnotetext{
${ }^{3}$ It was brought to my attention by H. Koch that, in the paper of N. Nadirashvili [Na], very interesting results related to stationary Euler equations were proved, in two dimensions, which are related partly to the current note (see $\mathrm{Na}$ Theorem 2.1]). In particular, Nadirashvili showed that the solutions of the problem in this paper, and their level surfaces, exhibit nice regularity properties at the points of nonvanishing gradient. This, seen in the light of free boundary analysis, might not be surprising, because once the free boundary has some regularity, one may bootstrap a higher regularity as done by V. Isakov, and later and independently by D. Kinderleherer and L. Nirenberg; see [PSU, Notes, p. 131], for a detailed account of this.
} 
Question. Let $u$ be a solution of the semilinear problem (11), with $f(u)$ continuous. Would this be enough to show that $u$ has continuous second derivatives? If this would not be true, then what if one adds the extra condition $f^{\prime} \geq-C 4$

It is quite tempting to stretch the above question further and ask whether $u$ has higher regularity whenever $f$ has; e.g. what are the conditions on $f(u)$ (beyond classical thresholds of $f^{(m-2)}$ being Dini) to ensure $D^{m} u(m \geq 2)$ to be bounded, or continuous?

In this regard, other aspects to look at are the case of systems of the type $\Delta \mathbf{u}=\mathbf{f}(|\mathbf{u}|)$ (bold text denotes vectors), or equations with lower regularities of the right-hand side like $\Delta u= \pm \log |u|$; the optimal regularity of solutions of the latter equation should be below $C^{1,1}$, due to the log-term (see QS ).

In the lines of the above discussions, it is noteworthy that semilinear problems involving the gradient have also been partly in focus. The well-known gradient constraint problem [Ev], as well as the so-called superconductivity problem [CSal, CSS] treats two different types of such problems. One may very generally ask for problems of the form $\Delta u=f(|\nabla u|)$, and how do solutions behave?

1.3. Quasilinear type operators. Another direction to develop the theory would be the case of divergence type operators of the form

$$
\operatorname{div}(f(x, u) \nabla u)=0,
$$

with conditions on $f$ to ensure the regularity of $D u$; here $0<\lambda \leq f \leq \Lambda<\infty$, to keep uniformity of the ellipticity. If $f$ is $C^{m, \alpha}$ (in both variables), then from the classical theory it follows that $u$ is $C^{m+1, \alpha}$. One can obviously push this to Dini continuity for $f^{(m)}$, and obtain continuity for $D^{m+1} u$. The classical theory also gives that, for bounded $f$, the solution $u$ is $C^{\beta}$ for some unknown $\beta \in(0,1)$. The constant $\beta$ can be improved ( $\beta \nearrow 1$ ) by asking $|f-1|$ to be small. This was done by a perturbation technique in $[\mathrm{CP}]$.

To be concrete, let us put forward the following question. Suppose $f(x, u)$ is continuous, but has less regularity than Dini in the $u$ variable, and is Dini in $x$. Can one claim that the solutions of (2) are Lipschitz?

As an example, consider the case where $f(u)=1+H(u)$, where $H$ is the Heaviside function. Then $v(x)=2 u^{+}-u^{-}$, where $u$ solves equation (2) and $u^{ \pm}:=\max ( \pm u, 0)$, is harmonic in $B_{1}$. Hence $\nabla v$ is bounded, and the bound depends on the supremum norm of $v$, and hence that of $u$. In particular $u$ is uniformly Lipschitz, with Lipschitz norm depending on $\|u\|_{\infty}$. One can argue similarly for $f(u)=2-H(u)$, by considering $v(x)=u^{+}-2 u^{-}$.

In (2), if $f$ is independent of $x$, one may use a simple argument 5 by considering $F$ (with $F^{\prime}=f$ ), and then (2) can be written as $\Delta F(u)=0$. Thus, $F(u)$ is $C^{\infty}$, and $F^{\prime}=f>\lambda$, so it has an inverse, and hence $u=F^{-1} F(u)$, with $F$ Lipschitz, i.e., $F^{-1}$ is Lipschitz. It follows that $u$ is Lipschitz. This cute idea does not apply (in general) when $f$ has $x$-dependency, and $f(x, u)$ has only $C^{\alpha}$-regularity in $x$; it works well if $f(x, u)$ is $C^{1, \alpha}$ in $x$-variable.

Notwithstanding this, one can derive a simple argument that implies at least $C^{\alpha}$-regularity (for any $\alpha<1$ ) in the case when $f(x, u)$ is continuous in $x$, and has right and left hand side limits (in the $u$-variable) at its discontinuity points. Let now $A^{ \pm}=$ $\lim _{u \rightarrow \pm 0} f(x, u)$, let $z$ be any point in $B_{1 / 2}$, and assume $u(z)=0$ (the nonzero points can be reduced to this by considering a translation in $f$ ).

Next, one considers a contradictory blow up argument (as done in [KLS] for a perturbation problem) and works with the scaled function $\widetilde{u}_{j}(x):=u_{j}\left(r_{j} x+z^{j}\right) / j r_{j}$, where

\footnotetext{
${ }^{4}$ As remarked in the earlier footnote, see N. Nadirashvili's paper $\mathrm{Na}$. for a similar discussion in this regard.

${ }^{5}$ This was kindly pointed out to me by Herbert Koch.
} 
the $z^{j}, u_{j}$, and $r_{j}$ are running sequences that contradict the statement in a way that

$$
\sup _{B_{R}}\left|\widetilde{u}_{j}\right| \leq C R^{\alpha}
$$

for all $R \geq 1$, and with equality for $R=1$,

$$
\sup _{B_{1}}\left|\widetilde{u}_{j}\right|=C .
$$

Then, the conclusion is that $u_{j}$ converges, for a subsequence, to a solution $u_{0}$ of

$$
\operatorname{div}\left[\left(A^{+} \chi_{\left\{u_{0}>0\right\}}+A^{-} \chi_{\left\{u_{0}<0\right\}}\right) \nabla u_{0}\right]=0 \text { in } \mathbb{R}^{n} .
$$

Invoking Liouville's theorem 6 along with (3) one can conclude that $u_{0}$ must be identically constant, and hence zero (since $u_{j}(0)=0$ ). This obviously contradicts the equality part of (3) for $R=1$.

It is not obvious how a proof for a possible Lipschitz regularity would proceed without any further technical tools. Let us see how one can try to obtain some information, for possible advancement in the theory. Since divergence type operators require Dinicontinuity of the coefficients to produce $C^{1}$ solutions, one may start by assuming that the function $f$ has smoothness to the right and left hand sides of a singular point. More exactly, assume that $f(x, u)$ has a jump at $u=0$, but is smooth otherwise. Let also $f^{ \pm}(x, u)=f(x, u)$ for $\pm u>0$. Then

$$
\operatorname{div}(f(x, u) \nabla u)=\operatorname{div}\left[\left(f^{+}(x, u) \chi_{\{u>0\}}+f^{-}(x, u) \chi_{\{u<0\}}\right) \nabla u\right],
$$

which can be simplified to

$$
\operatorname{div}(f(x, u) \nabla u)=\operatorname{div}\left[\left(a(x)+b(x) \chi_{\{u<0\}}\right) \nabla u\right],
$$

where $a(x)=f^{+}(x, u(x))$, and $b(x)=f^{-}(x, u(x))-f^{+}(x, u(x))$, with $a, b$ being smooth functions, say Dini-continuous.

The reformulated form of the problem at hand may now make it possible for us to try a monotonicity-function argument, as done for the previous case. There is so far no such type of monotonicity function for a quasilinear equation, but the author believes that similar arguments used by previous authors for perturbed operators (see $\mathrm{MP}$ ) should work here as well, provided $a, b$ are sufficiently regular. This is a subject for investigation in [KLS], and I shall not touch upon it here. As a conclusion to the discussion, the following conjecture can be put forward.

Conjecture 1.4. Any (weak/viscosity) solution u of the semilinear elliptic equation

$$
\operatorname{div}(f(x, u) \nabla u)=0 \text { in } B_{1},
$$

where $f$ is Dini continuous in the $x$-variable and has only finitely many discontinuities in the u-variable and uniform $C^{\alpha}$ regularity to the right and left of the discontinuities, should have universally bounded derivatives in $B_{1 / 2}$, with norms depending on $\|u\|_{\infty}$, the ellipticity constants, and the space dimension.

It is plausible that the assumption of having a finite number of discontinuities is superfluous, and the mere assumption that $f(x, u)$ has left- and right-hand side Dini smoothness (in the $u$-variable) at every point should suffice to conclude Lipschitz regularity for the solution. Also it seems reasonable to think that a more complicated structure as $\mathbf{f}(x, u, p)$ (where $\mathbf{f}$ is now a vector) with reasonable regularity in $x$ and $p$,

\footnotetext{
${ }^{6}$ Here, the Liouville type argument goes as follows: For each $z \in \mathbb{R}^{n}$, consider $v_{R}(x):=$ $u_{0}(R x+z) / R^{\alpha}$, which again is a solution in $B_{1}(0)$ and is bounded by (3). In particular, $v_{R}$ is Lipschitz, by the discussions above for the constant coefficient case. Hence, $\sup _{B_{r}(0)}\left|v_{R}(x)\right| \leq C r$, i.e., $R^{\alpha}\left|v_{R}(0)\right|=\left|u_{0}(z)\right| \leq C r R^{\alpha}$. Choosing $r=1 / R$, and letting $R$ tend to infinity, one arrives at $u_{0}(z)=0$.
} 
and a similar one as in the conjecture in $u$, would give Lipschitz regularity for solutions to $\operatorname{div} \mathbf{f}(x, u, \nabla u)=0$.

Remark 1.5. Our method of approach, with scaling and blow-up technique utilized above, works also for the operator $\operatorname{div} \mathbf{f}(x, u, \nabla u)=0$, but it implies only regularity below Lipschitz.

1.4. Regularity of level sets (heuristics). The regularity of the level sets of a solution of any of the above problems is also an interesting issue. For several concrete examples, this has been studied earlier (see [PSU] for some examples and also references). To look into the theory of regularity, one can consider problem (11) at points where the gradient of the solution vanishes (otherwise implicit function theory gives regularity).

Let us come back to pointwise values of $f$ at discountinuty points, by setting

$$
\pm B^{ \pm}=\lim _{s \rightarrow \pm s_{0}} f(s) \text {. }
$$

Now, if $B^{+}+B^{-} \geq 0$, then $f^{\prime}\left(s_{0}\right) \geq 0$, and this could be seen as a structural condition for $C^{1,1}$-regularity, for $u$. It has been established in the literature that the theory for $C^{1,1}$-solutions (see [PSU]) and that for non- $C^{1,1}$-solutions (see [ASW] differ substantially.

Nondegeneracy of $u$ and Lebesgue measure of level sets. The nondegeneracy (for at least one phase) for $u$ will follow in general from the assumption $B^{+}+B^{-} \geq \lambda_{0}>0$. Indeed, this implies that $\max \left(B^{+}, B^{-}\right) \geq \lambda_{0} / 2>0$. One may assume that $B^{+} \geq \lambda_{0} / 2$, otherwise one looks at the function $w=s_{0}-u$, with $\Delta w=-f\left(s_{0}-w\right)=: \widetilde{f}(w)$, so that $\tilde{f}^{\prime}(w)=f^{\prime}(u)$.

Next, consider the function $v=u-s_{0}$, where $s_{0}=u\left(x^{0}\right)$. Then $v$ satisfies $\Delta v=g(v)$, with $g(v):=f\left(v+s_{0}\right)$, and obviously $g(v)>\lambda_{0}$ on the set $\{v>0\}$. Hence the standard nondegeneracy argument applies to this setting (see [PSU]).

Along with the optimal $C^{1,1}$ regularity, this implies (as was done for the obstacle problem) that the level surface has zero measure (cf. the proof for Problem $C$ in [PSU]).

Below, I shall present different possible situations that may arise when we study the regularity of level sets. I shall specify these cases and give some hint of how a possible study might be carried out. These are all basically open problems, and may be picked up by an interested reader for a detailed analysis.

Case 1. $B^{+}=B^{-}=0$.

This case is complicated, as it means that locally the solution $u$ is harmonic at the origin. The behavior of the level set around such a point can be of any type. One would then need to have more information about how fast $f(x, u)$ tends to zero in $u$; e.g., if $f(x, u) \approx b(x)|u|^{a}$ (with $b \geq c>0$ ), then one needs to use approaches that have been developed (partly) by H. W. Alt and D. Philips (see AP ). If also $b(x)$ becomes zero, then there is one more difficulty to take care of. Such type of problems were also looked at in $[\mathrm{CF}]$; cf. also a recent work in this direction $\mathrm{Ag}$.

A further line of directions that is being developed is the case where degeneracy is along a given surface (or a curve in 2-space dimension). I refer the reader to the recent paper [Ye] by K. Yerissian [Ye]. I shall not discuss this case further.

Case 2. $B^{+}>B^{-}=0$ (or the reverse $B^{-}>B^{+}=0$ ).

This is reminiscent of the obstacle problem, but with a possibility that $u^{-} \neq 0$, and that under a scaling it will disappear (see [MP2] for a particular case of this problem). To have a closer look at this case, I assume that the origin is on the free boundary with (as above) $u(0)=s_{0}=0$, and $\nabla u(0)=0$ (otherwise the free boundary is smooth close to this point, due to the implicit function theorem). Next, a scaling $u_{j}(x)=u\left(r_{j} x\right) / r_{j}^{2}$ will 
converge (for a subsequence) to a global solution $u_{0}$ satisfying

$$
\Delta u_{0}=B^{+} \chi_{\left\{u_{0}>0\right\}}, \quad \nabla u_{0}(0)=0 .
$$

Now, to avoid singular (cusp) points (these points occur for special cases of $f$ ), one can assume that the level surface at the origin has an a priori regularity, in either geometric terms, such as the set $\{u \leq 0\}$ has a thickness in such a way that it prevails under the blow up and hence $\left\{u_{0} \leq 0\right\}$ has nonempty interior, or in terms of the ACF monotonicity function $\phi 7$ such that $\lim _{r \rightarrow 0} \phi(r, u, 0)=0$. Since $\phi(r) \geq 0$, and it is upper semicontinuous, we conclude that the set of points with the minimum value of $\phi$ (lowenergy points) must be an open set. This is an important observation in the regularity theory, and leads to the fact that it would suffice to study only points of low-energy level, from a regularity point of view. Indeed, in a blow-up/scale regime, such points lead to the so-called half-space solutions (i.e., one-dimensional solutions), hence providing us with a fixed direction for monotonicity of the solution, and one may then try to prove the Lipschitz regularity of the free boundary as it was done for the obstacle type problems; see [PSU].

Case 3. $\min \left(B^{+}, B^{-}\right)>0$.

In this case, a blow up $u_{0}$ satisfies

$$
\Delta u_{0}=B^{+} \chi_{\left\{u_{0}>0\right\}}-B^{-} \chi_{\left\{u_{0}<0\right\}}
$$

which is the two-phase free boundary problem studied in [SUW1- SUW2], see also [PSU]. The only global solution is one-dimensional, which after rotation is given by $u_{0}=$ $B^{+}\left(x_{1}^{+}\right)^{2} / 2-B^{-}\left(x_{1}^{-}\right)^{2} / 2$. Hence, one may now apply the directional monotonicity argument 8 to show that $D_{e} u(x) \geq 0$ for $e \approx e_{1}$ and $x \in B_{r}$ for some sufficiently small $r$. This proves that the free boundary is Lipschitz. By changing the direction of the vector $e$ in the above and keeping $e \cdot e_{1}>0$, one can then show it is $C^{1}$. One can actually prove it is uniformly $C^{1}$, but I will not discuss this here, as it requires a more complicated analysis.

Case 4. $B^{+}>0>B^{-}, B^{+}+B^{-} \geq 0$.

In this case $u^{-}$could possibly be degenerate, and in a blow-up regime one has

$$
\Delta u_{0}=B^{+} \chi_{\left\{u_{0}>0\right\}}-B^{-} \chi_{\left\{u_{0}<0\right\}},
$$

with $u^{-}$possibly zero. This somehow falls under Case 2 above, but might need more care.

Case 5. $B^{+}>0>B^{-}, B^{+}+B^{-}<0$.

In this case the solution is not necessarily $C^{1,1}$, and hence the set of points $\{\nabla u=0\}$ has to be split into two classes, one with quadratic growth and one with nonquadratic growth. The latter was studied in [ASW] (in 3 dimensions), and the former was looked at in a very particular case by R. Monneau and G. S. Weiss in MW. There are still many unanswered questions for this case.

1.5. Regularity close to the fixed boundary. When the setting of the problems discussed above are done in presence of a fixed boundary (as in [SU]), one cannot apply the monotonicity function at the boundary points because the function is not $C^{2}$ there; it is at least not $C^{2}$ at the points where the free and fixed boundary touch, and this is needed to apply the monotonicity lemma argument (see [Sh]).

\footnotetext{
${ }^{7}$ The reader may consult $\mathrm{PSU}$ for the definition of the ACF monotonicty function, and how it is used.

${ }^{8}$ Directional monotonicity refers to showing that $\operatorname{Cr} D_{e} u-u \geq 0$ in $B_{r}$ for small $r$ and $e \cdot e_{1}>0$. This means that $u$ is monotone in all directions not orthogonal to the $x_{1}$-axis (in the rotated system chosen in the argument above), and in a ball depending on the value of $e_{1}$.
} 
A first estimate to obtain is that for the tangential directions to the fixed boundary. I assume the boundary to be a flat surface, say $\left\{x_{n}=0\right\}$; so more exactly I consider

$$
\Delta u=f(u) \text { in } B_{1}^{+}(0), \quad u\left(x^{\prime}, 0\right)=0,
$$

where $x^{\prime}=\left(x_{1}, \ldots, x_{n-1}\right)$. Again I ignore the boundary values on the sphere. Now, considering $\left(D_{e_{j}} u\right)^{ \pm}$for $j=1, \ldots,(n-1)$, one sees that they have Laplacian bounded from below when $f^{\prime} \geq-c$, and they are zero on $\left\{x_{n}=0\right\} \cap B_{1}$. Hence we can control them by the barrier $C x_{n}-c x_{n}^{2} / 2$ (in a sufficiently small ball around the origin), whence

$$
\sup _{B_{r}^{+}(0)}\left|\left(D_{e_{j}} u\right)\right| \leq C_{0} r,
$$

or more accurately $\left(D_{e_{j}} u\right)^{ \pm}(x) \leq C_{0} x_{n}-c x_{n}^{2} / 2$.

Now we consider the application of the monotonicity function to $\left(D_{e_{j}} u\right)^{ \pm}$at the interior points $z$ with $z_{n}>0$ where the second derivatives exist. Then the radius $r$ in the monotonicity function can not exceed the value $z_{1}$. This would mean that $\left|\nabla D_{\tau} u(z)\right| \leq C_{r}(\tau \perp \nabla u(z))$ with $C_{r}=C r^{-n}\left\|\nabla D_{\tau} u\right\|_{L^{2}\left(B_{r}(z)\right)}$. From the PDE one also obtains the estimate $\left\|D_{\eta \eta}^{2} u(z)\right\| \leq C_{r}+\sup _{B_{1}}|f|$, where $\eta=\nabla u(z) /|\nabla u(z)|$. Hence, $\left\|D^{2} u(z)\right\| \leq C^{\prime} r^{-n}\left\|\nabla D_{\tau} u\right\|_{L^{2}\left(B_{r}(z)\right)}$ for some $C^{\prime}$ and all $r<z_{1}$. Combining this with (5) and the methods of Lemma 6.3 in [SU], one obtains the estimate on the second derivatives at interior points.

1.6. Tangential touch between the free and fixed boundary. A systematic analysis, as hinted in Cases 1-5 above for local regularity, can also be carried out for the behavior of the zero level sets close to the fixed boundary. In general, a tangential touch between $\partial\{u>0\}$ and the fixed boundary $\left\{x_{n}=0\right\}$ is expected. It would probably need a deeper study to prove that $\partial\{u>0\}$ is locally a $C^{1}$-graph. It is left to the interested reader to carry out the details, and enjoy the surprising difficulties the problem may offer.

\section{$\S 2$. Parabolic case}

A similar theory for parabolic problems can be done, first, by proving a bound for the time derivative. This is possible due to the monotonicity of $f 9$ To see this, one sets $u_{\tau}(X):=[u(x, t+\tau)-u(X)]$ with $X=(x, t)$ and applies the heat operator to $u_{\tau}^{ \pm}$to find out that

$$
\Delta u_{\tau}^{ \pm}-\partial_{t} u_{\tau}^{ \pm}=h_{\tau}(X) u_{\tau}^{ \pm} \geq-C u_{\tau}^{ \pm},
$$

where $h_{\tau}(X)=[f(u(x, t+\tau))-f(u(X))] /\left[u_{\tau}\right] \geq-C$. Now, the $u_{\tau}^{ \pm}$being sub caloric, one may apply the maximum principle for weak sub-solutions (see [Li, Theorem 7.21]) for the right-hand side $-C u_{\tau}^{ \pm}$to obtain

$$
\sup _{Q_{1 / 2}} u_{\tau}^{ \pm} \leq C^{\prime}\left[\left\|u_{\tau}^{ \pm}\right\|_{L^{p}\left(Q_{1}\right)}+\left\|C u_{\tau}^{ \pm}\right\|_{L^{n+1}\left(Q_{1}\right)}\right]
$$

where $Q_{1}$ is the parabolic cylinder; more precisely, we define

$$
Q_{r}(X)=B_{r}(x) \times\left(-r^{2}+t, r^{2}+t\right) .
$$

Now, letting $p \geq n+1$ and assuming that the solution has time derivatives in $L^{p}$, we can let $\tau$ tend to zero to obtain bounds on the time derivative:

$$
\sup _{Q_{1 / 2}}\left|\partial_{t} u\right| \leq C^{\prime \prime}\left\|\partial_{t} u\right\|_{L^{p}\left(Q_{1}\right)}
$$

\footnotetext{
${ }^{9}$ The idea here is based on Ural'tseva's paper [Ur], cf. also [SUW].
} 
where $C^{\prime \prime}$ depends on $C$, the space dimension and other ingredients. Observe that in this argument there is no need for $u$ to have good regularity a priori. Hence, $f \in L^{p}$ would suffice.

Now the argument for bounds on the second spatial derivatives involves the monotonicity formula, which in part asks the functions $D_{e} u$ to be zero at the center for the monotonicity function $Z=(z, s)$. For this, one chooses $e$ to be orthogonal to the spatial gradient $\nabla u(Z)$ (after rotation, this can be assumed to be $e_{1}$ ) and then, after applying the monotonicity function, one arrives at uniform bounds for $\nabla D_{e} u(Z)$. In the case where $\nabla u(Z) \neq 0$, one obtains bounds on $D_{j k} u$ for all $j, k$, but not for $j=k=1$. Bounds on $D_{11} u$ are then achieved by using the equation

$$
D_{11} u=-D_{22} u-\cdots-D_{n n} u+\partial_{t} u+f(u),
$$

which is bounded due to bounds for $f, D_{i i} u$ for $i=2, \ldots, n$, and $\partial_{t} u$ (due to (6) ). This implies the following parabolic counterpart of Theorem 1.2

Theorem 2.1. Suppose that $u \in W_{x}^{2, p} \cap W^{1, p}\left(Q_{1}\right)(p \geq n+1)$ solves

$$
\Delta u-\partial_{t} u=f(u) \text { in } Q_{1},
$$

with $f^{\prime} \geq-C$. Then for any point $Z \in Q_{1 / 2}$ and any directional vector $e \perp \nabla u(Z)$, we have

$$
\lim _{r \rightarrow 0} \frac{1}{r^{n+2}} \int_{Q_{r}(Z)}\left|\nabla D_{e} u\right|^{2} \leq C^{\prime}\left\|\nabla D_{e} u\right\|_{L^{2}\left(Q_{1}\right)}^{2} .
$$

In particular, at any point $Z=(z, s)$ we have $\left|D_{e} u(x, s)-D_{e} u(Z)\right| \leq C^{\prime \prime}|x-z|$ for all $e \perp \nabla u(Z)$. If $\nabla u(Z)=0$, then $|\nabla u(x, s)-\nabla u(Z)| \leq C^{\prime \prime}|x-z|$.

Any attempts for replacing the Laplacian with a divergence, or nondivergence type operators (with $C^{\alpha}$-coefficients) have so far failed. To formulate a conjecture (in this regard), let the matrix $\left(a_{i j}\right)$ have standard uniform ellipticity as well as $C^{\alpha}$ entries.

Thus, we conjecture the following.

Conjecture 2.2. Any (weak/viscosity) solution $u$ of the uniformly elliptic semilinear parabolic equation

$$
\sum_{i j} a_{i j} D_{i j} u-\partial_{t} u=f(x, t, u) \text { in } Q_{1}
$$

where $f_{u}^{\prime} \geq-c_{0}$ and $f$ is $C^{\alpha}$ in the $(x, t)$-variables, should have universally bounded second spatial and first time derivatives in the cylinder $Q_{1 / 2}$, with norms depending on $\|u\|_{\infty}, c_{0}, c_{1}$, and the space dimension.

The same question can be asked for elliptic problems. In the case of the Laplacian, I required $f$ to be Lipschitz in the $x$-variable, but that (probably superfluous) requirement depends on the monotonicity function tool, rather than anything else.

A similar and systematic analysis of the free boundary (as discussed in the elliptic case) can be done for the parabolic setting. However, so far there has been only little work in this regard. It is noteworthy that the supremum norm estimate for the time derivative can also be carried out as in the case of Laplacian, as discussed in the paragraph preceding the above conjecture.

2.1. Parabolic quasilinear problems. Now I consider the parabolic counterpart of the quasilinear problem (2),

$$
\partial_{t} u-\operatorname{div}(f(u) \nabla u)=0,
$$

where $f$ is as in (2). It is apparent that the simple ideas (of looking at $\partial_{t} u-\Delta(F(u))=$ 0 ) discussed in the elliptic case do not apply here, and one needs to introduce new techniques. To my best knowledge, this problem seems not to be covered by classical 
methods. John Andersson gave me a nice idea to reformulate this by letting $v=F(u)$ and applying the heat operator to this to arrive at $\partial_{t} v-g(v) \Delta v=0$, where $g(v)=$ $f(u)=f\left(F^{-1}(v)\right)$. This changes the problem, though does not necessarily simplify it. It is clear that the scaling argument and a Liouville type theorem for the limit function would imply (as discussed in the elliptic case) that the solutions are $C_{x}^{\alpha} \cap C_{t}^{\alpha / 2}$ locally, uniformly in the domain of definition for the equation. Here any $\alpha<1$ works.

Conjecture 2.3. Any (weak/viscosity) solution $u$ of the semilinear parabolic equation

$$
\partial_{t} u-\operatorname{div}(f(x, u) \nabla u)=0 \text { in } Q_{1},
$$

where $f(x, u)$ is Dini in $x$, has only finitely many number discontinuities in the u-variable, and is uniformly $C^{\alpha}$ regular to the right and left of the discontinuities, should have uniformly bounded $x$-derivatives in $Q_{1 / 2}$, with norms depending on $\|u\|_{\infty}$, ellipticity, and the space dimension.

\section{REFERENCES}

[Ag] A. Aghajani, A two-phase free boundary problem for a semilinear elliptic equation, Bull. Iranian Math. Soc. 40 (2014), no. 5, 1067-1086. MR3273825

[AP] H. W. Alt and D. Phillips, A free boundary problem for semilinear elliptic equations, J. Reine Angew. Math. 368 (1986), 63-107. MR850615

[ACF] H. W. Alt, L. A. Caffarelli, and A. Friedman, Variational problems with two phases and their free boundaries, Trans. Amer. Math. Soc. 282 (1984), no. 2, 431-461. MR732100

[ASW] J. Andersson, H. Shahgholian, and G. S. Weiss, On the singularities of a free boundary through Fourier expansion, Invent. Math. 187 (2012), no. 3, 535-587. MR2891877

[AW1] J. Andersson and G. S. Weiss, Cross-shaped and degenerate singularities in an unstable elliptic free boundary problem, J. Differential Equations 228 (2006), no. 2, 633-640. MR2289547

[CP] L. A. Caffarelli and I. Peral, On W1,p estimates for elliptic equations in divergence form, Comm. Pure Appl. Math. 51 (1998), no. 1, 1-21. MR1486629

[EP] A. Edquist and A. Petrosyan, A parabolic almost monotonicity formula, Math. Ann. 341 (2008), no. 2, 429-454. MR2385663

[Ev] L. C. Evans, A second-order elliptic equation with gradient constraint, Comm. Partial Differential Equations 4 (1979), no. 5, 555-572. MR529814

[CF] L. A. Caffarelli and A. Friedman, The free boundary in the Thomas-Fermi atomic model, J. Differential Equations 32 (1979), no. 3, 335-356. MR535167

[CJK] L. A. Caffarelli, D. Jerison, and C. E. Kenig, Some new monotonicity theorems with applications to free boundary problems, Ann. of Math. (2) 155 (2002), no. 2, 369-404. MR.1906591

[CSal] L. A. Caffarelli and J. Salazar, Solutions of fully nonlinear elliptic equations with patches of zero gradient: existence, regularity and convexity of level curves, Trans. Amer. Math. Soc. 354 (2002), no. 8, 3095-3115. MR1897393

[CSS] L. Caffarelli, L. Salazar, and H. Shahgholian, Free-boundary regularity for a problem arising in superconductivity, Arch. Ration. Mech. Anal. 171 (2004), no. 1, 115-128. MR2029533

[KLS] S. Kim, K. Lee, and H. Shahgholian, A free boundary arising from the jump of conductivity. (to appear)

[Li] G. M. Lieberman, Second order parabolic differential equations, World Sci. Publ. Co., Inc., River Edge, NJ, 1996. MR 1465184

[MP] N. Matevosyan and A. Petrosyan, Almost monotonicity formulas for elliptic and parabolic operators with variable coefficients, Comm. Pure Appl. Math. 64 (2011), no. 2, 271-311. MR2766528

[MP2] _ Two-phase semilinear free boundary problem with a degenerate phase, Calc. Var. Partial Differential Equ. 41 (2011), no. 3-4, 397-411. MR2796237

[MW] R. Monneau and G. S. Weiss, An unstable elliptic free boundary problem arising in solid combustion, Duke Math. J. 136 (2007), no. 2, 321-341. MR.2286633

[Na] N. Nadirashvili, On stationary solutions of two-dimensional Euler equation, Arch. Ration. Mech. Anal. 209 (2013), no. 3, 729-745. MR3067825

[PSU] A. Petrosyan, H. Shahgholian, and N. N. Ural'tseva, Regularity of free boundaries in obstacletype problems, Grad. Stud. Math., vol. 136, Amer. Math. Soc., Providence, RI, 2012. MR2962060

[QS] O. S. de Queiroz and H. Shahgholian, A free boundary problem with log-term singularity. (to appear) 
[Sh] H. Shahgholian, $C^{1,1}$ regularity in semilinear elliptic problems, Comm. Pure Appl. Math. 56 (2003), no. 2, 278-281. MR1934623

[SU] H. Shahgholian and N. N. Ural'tseva, Regularity properties of a free boundary near contact points with the fixed boundary, Duke Math. J. 116 (2003), no. 1, 1-34. MR.1950478

[SUW1] H. Shahgholian, N. N. Ural'tseva, and G. S. Weiss, Global solutions of an obstacle-problem-like equation with two phases, Monatsh. Math. 142 (2004), no. 1-2, 27-34. MR2065019

[SUW2] _ The two-phase membrane problem - regularity of the free boundaries in higher dimensions, Int. Math. Res. Not. IMRN 2007, no. 8, Art. ID rnm026. MR2340105

[SUW3] _ A parabolic two-phase obstacle-like equation, Adv. Math. 221 (2009), no. 3, 861-881. MR2511041

[TZ] E. V. Teixeira and L. Zhang, A local parabolic monotonicity formula on Riemannian manifolds, J. Geom. Anal. 21 (2011), no. 3, 513-526. MR2810841

[Ur] N. N. Ural'tseva, Boundary estimates for solutions of elliptic and parabolic equations with discontinuous nonlinearities, Nonlinear Equations and Spectral Theory, Amer. Math. Soc. Transl. Ser. 2, vol. 220, Amer. Math. Soc., Providence, RI, 2007, 235-246. MR2343613

[Ye] K. Yerissian, Nondegeneracy in the obstacle problem with a degenerate force term, Interfaces Free Bound. 17 (2015), no. 2, 233-244. MR3391970

Department of Mathematics, Royal Institute of Technology, 10044 Stockholm, Sweden

E-mail address: henriksh@kth.se

Received 2/MARCH/2015

Originally published in English 